\title{
Otolaryngologic Disorder
}

National Cancer Institute

\section{Source}

National Cancer Institute. Otolaryngologic Disorder. NCI Thesaurus. Code C118420.

A non-neoplastic or neoplastic disorder that affects the ears, nose, paranasal sinuses,

oral cavity, or throat. 